PROCEEDINGS OF THE

AMERICAN MATHEMATICAL SOCIETY

Volume 128, Number 7, Pages 2187-2190

S 0002-9939(99)05238-7

Article electronically published on October 29, 1999

\title{
COMPACTIFICATIONS AND UNIVERSAL SPACES IN EXTENSION THEORY
}

\author{
ALEX CHIGOGIDZE
}

(Communicated by Alan Dow)

\begin{abstract}
We show that for each countable simplicial complex $P$ the following conditions are equivalent:

- $P \in A E(X)$ iff $P \in A E(\beta X)$ for any space $X$.

- There exists a $P$-invertible map of a metrizable compactum $X$ with $P \in$ $A E(X)$ onto the Hilbert cube.
\end{abstract}

\section{INTRODUCTION}

The following two problems are central in extension theory [6] (see also [4]).

Problem A ( 7 , Problem 5]). Characterize $\mathrm{CW}$-complexes $P$ such that for any space $X$ with $P \in A E(X)$ there exists a compactification $b X$ of $X$ such that $P \in A E(b X)$.

Problem B ([7, Problems 6, 7]). Characterize CW-complexes $P$ such that the class

$$
\{X: X \text { is a separable metrizable space with } P \in A E(X)\}
$$

has a universal space (compactum).

The first example of a space with $K(\mathbb{Z}, n) \in A E(X)$ and $K(\mathbb{Z}, n) \notin A E(\beta X)$ (in other words $\operatorname{dim}_{\mathbb{Z}} X<\operatorname{dim}_{\mathbb{Z}} \beta X$ ) was constructed by A. N. Dranishnikov [5] (see also [10]). There even exist spaces with $K(\mathbb{Z}, n) \in A E(X)$ and $K(\mathbb{Z}, n) \notin A E(b X)$ for any compactification $b X$ of $X$ [9]. It follows from [7, Corollary 2.5(b) and its proof] that for a finitely dominated complex $P$ the class indicated in problem $\mathrm{B}$ has a universal compactum. It is important to emphasize that a universal compactum in this case can be produced (see the proof of [7] Theorem 2.3]) as the domain of a $P$-invertible map the range of which is the Hilbert cube $\mathbb{I}^{\omega}$. Various results suggest and several authors have observed (see, for instance, [8, Remark], [7, p.1657]) that there seems to be a connection between the existence of "dimension" preserving compactifications and the existence of universal elements in the class of metrizable compacta of a given "dimension".

Below we consider stronger versions of the above problems.

Received by the editors April 14, 1998 and, in revised form, August 25, 1998.

1991 Mathematics Subject Classification. Primary 55M10; Secondary 54F45.

Key words and phrases. Compactification, universal space, cohomological dimension.

The author was partially supported by NSERC research grant.

(C)2000 American Mathematical Society 
Problem $\mathbf{A}^{\prime}$. Characterize connected locally compact simplicial complexes 1 such that $P \in A E(X)$ iff $P \in A E(\beta X)$ for any space $X$.

Problem $\mathbf{B}^{\prime}$. Characterize connected locally compact simplicial complexes $P$ such that there exists a $P$-invertible map $f: X \rightarrow \mathbb{I}^{\omega}$ where $X$ is a metrizable compactum with $P \in A E(X)$.

Below we show (Theorem 2.1) that Problems $\mathrm{A}^{\prime}$ and $\mathrm{B}^{\prime}$ are equivalent.

\section{Results}

All spaces are assumed to be Tychonov (i.e. completely regular and Hausdorff). All maps are continuous. II denotes a closed interval. All simplicial complexes are connected and locally compact. If $X$ is a normal space, we say that $P$ is an absolute extensor of $X$ (and write $P \in A E(X)$ ) if for each closed subspace $Y$ of $X$ any map $f: Y \rightarrow P$ has a continuous extension $\bar{f}: X \rightarrow P$. An extension of this concept for non-normal spaces has been given in [3, Definition 3.1]. A map $f: X \rightarrow Y$ is $P$ invertible if for any map $g: Z \rightarrow Y$ with $P \in A E(Z)$ there exists a map $h: Z \rightarrow X$ such that $g=f h$.

Theorem 2.1. Let $P$ be a Polish ANR-space. Then the following statements are equivalent:

(a) $P \in A E(\beta X)$ whenever $X$ is a space with $P \in A E(X)$.

(b) $P \in A E(\beta X)$ whenever $X$ is a normal space with $P \in A E(X)$.

(c) $P \in A E\left(\beta\left(\oplus\left\{X_{t}: t \in T\right\}\right)\right)$ whenever $T$ is an arbitrary indexing set and $X_{t}$, $t \in T$, is a separable metrizable space with $P \in A E\left(X_{t}\right)$.

(d) $P \in A E\left(\beta\left(\oplus\left\{X_{t}: t \in T\right\}\right)\right)$ whenever $T$ is an arbitrary indexing set and $X_{t}$, $t \in T$, is a Polish space with $P \in A E\left(X_{t}\right)$.

(e) There exists a P-invertible map $f_{P}: K_{P} \rightarrow \mathbb{I}^{\omega}$ where $K_{P}$ is a metrizable compactum with $P \in A E\left(K_{P}\right)$.

Proof. Implications (a) $\Longrightarrow(\mathrm{b}) \Longrightarrow(\mathrm{c}) \Longrightarrow$ (d) are trivial. Proof of implication (d) $\Longrightarrow$ (e) follows the proof of [3 Proposition 5.3]. Let $\mathcal{A}$ denote the set of all maps $\left\{r_{t}: t \in T\right\}$ such that domain $\operatorname{Dom}\left(r_{t}\right)$ is a Polish subspace of $\mathbb{I}^{\omega}, P \in$ $A E\left(\operatorname{Dom}\left(r_{t}\right)\right)$ and $\operatorname{Ran}\left(r_{t}\right) \subseteq \mathbb{I}^{\omega}$. Let $Y=\bigoplus\left\{\operatorname{Dom}\left(r_{t}\right): t \in T\right\}$. Clearly, $P \in$ $A E(Y)$. Consider also the map $r: Y \rightarrow \mathbb{I}^{\omega}$ which coincides with $r_{t}$ on $\operatorname{Dom}\left(r_{t}\right)$ for each $t \in T$. Let $\bar{r}: \beta Y \rightarrow \mathbb{I}^{\omega}$ be the unique continuous extension of $r$ to the Stone-Čech compactification $\beta Y$ of $Y$. By (d), $P \in A E(\beta Y)$. By [3, Theorem 4.4] and by the compactness of $\beta Y$, the latter is the limit space of a Polish spectrum $\mathcal{S}=\left\{Y_{\alpha}, q_{\alpha}^{\beta}, A\right\}$ consisting of metrizable compacta $Y_{\alpha}$ (compactness of $Y_{\alpha}$ follows from the fact that $q_{\alpha}(\beta Y)$ is dense in $Y_{\alpha}$, according to assmption made in [3. p. 201]) with $P \in A E\left(Y_{\alpha}\right)$. Write $\mathbb{I}^{\omega}=\prod\left\{\mathbb{I}_{n}: n \in \omega\right\}$ where $\mathbb{I}_{n}, n \in \omega$, denotes a copy of $\mathbb{I}$. Also let $\pi_{n}: \mathbb{I}^{\omega} \rightarrow \mathbb{I}_{n}, n \in \omega$, denote the corresponding projection. Since the spectrum $\mathcal{S}$ is factorizing, for each $n \in \omega$ there exist an index $\alpha_{n} \in A$ and a map $s_{n}: Y_{\alpha_{n}} \rightarrow \mathbb{I}_{n}$ such that $\pi_{n} \bar{r}=s_{n} q_{\alpha_{n}}$, where $q_{\alpha_{n}}: \beta Y \rightarrow Y_{\alpha_{n}}$ is the $\alpha_{n}$-th limit projection of the spectrum $\mathcal{S}$. Since $\mathcal{S}$ is a Polish spectrum (see [3, page 201]) there exists an index $\alpha \in A$ such that $\alpha \geq \alpha_{n}$ for each $n \in \omega$. Next consider the map

$$
s=\triangle\left\{s_{n} q_{\alpha_{n}}^{\alpha}: n \in \omega\right\}: Y_{\alpha} \rightarrow \prod\left\{\mathbb{I}_{n}: n \in \omega\right\},
$$

\footnotetext{
${ }^{1}$ We prefer to work with connected locally compact simplicial complexes because they are Polish ANR's and consequently results of [2, 3] apply.
} 
where $q_{\alpha_{n}}^{\alpha}: Y_{\alpha} \rightarrow Y_{\alpha_{n}}, n \in \omega$, denotes the corresponding projection of the spectrum $\mathcal{S}$. It is easy to see that $\bar{r}=s q_{\alpha}$, where $q_{\alpha}: \beta Y \rightarrow Y_{\alpha}$ is the $\alpha$-th limit projection of the spectrum $\mathcal{S}$. It now suffices to let $K_{P}=Y_{\alpha}$ and $f_{P}=s$. Let us show that $s: Y_{\alpha} \rightarrow \mathbb{I}^{\omega}$ is indeed $P$-invertible. Since the spaces $Y_{\alpha}$ and $\mathbb{I}^{\omega}$ are Polish (even compact and metrizable), it suffices (according to [3. Proposition 5.2]) to consider only Polish spaces $Z$ in the definition of $P$-invertibility given above. Indeed, let $g: Z \rightarrow \mathbb{I}^{\omega}$ be a map defined on a Polish space $Z$ with $P \in A E(Z)$. We may as well assume that $Z \subseteq \mathbb{I}^{\omega}$. By the definition of $\mathcal{A}$, there is an index $t \in T$ such that $r_{t}=g$. Let $i_{t}: \operatorname{Dom}\left(r_{t}\right) \rightarrow Y$ denote the corresponding embedding. Clearly, $r_{t}=\bar{r} i_{t}$. Then the composition $h=q_{\alpha} i_{t}: Z \rightarrow Y_{\alpha}$ lifts the map $g$, i.e. $s h=g$.

(e) $\Longrightarrow$ (a). As in the proof of [3, Theorem 5.13] (see also [2, Section 6.2] where the case $P=S^{n}$ is considered) one shows that for any uncountable cardinal number $\tau$ there exists a $P$-invertible map $f=f_{P, \tau}: K_{P, \tau} \rightarrow \mathbb{I}^{\tau}$, where $K_{P, \tau}$ is a compactum of weight $\tau$ such that $P \in A E\left(K_{P, \tau}\right)$ and $\mathbb{I}^{\tau}$ denotes the Tychonov cube of weight $\tau$ (for $\tau=\omega$ the existence of such a map is guaranteed by condition (e); note also that an $A E(0)$-space of countable weight is Polish [2, Corollary 6.4.5]). Consider now a space $X$ with $P \in A E(X)$ and choose $\tau$ large enough so that $\beta X$ can be identified with a subspace of $\mathbb{I}^{\tau}$. Since the map $f$ is $P$-invertible there exists a map $g: X \rightarrow K_{P, \tau}$ such that $f g=\operatorname{id}_{X}$. Since $K_{P, \tau}$ is compact, the map $g$ admits a continuous extension $\bar{g}: \beta X \rightarrow K_{P, \tau}$. Since $f g=\operatorname{id}_{X}$ and since $\bar{g} \mid X=g$, it follows that $f \bar{g}=\operatorname{id}_{\beta X}$. In this situation it can easily be seen that $\bar{g}$ is an embedding. In other words, $\bar{g}(\beta X)$ is a topological copy of $\beta X$. Finally, since $P \in A E\left(K_{P, \tau}\right)$ it follows that $P \in A E(\beta X)$.

Corollary 2.2. Let $P$ be a finitely dominated connected locally compact complex. Then the following conditions are equivalent for any space $X$ :

(1) $P \in A E(X)$.

(2) $P \in A E(\beta X)$.

Proof. The implication (2) $\Longrightarrow$ (1) follows from [3, Proposition 6.8]. Let us prove the implication (1) $\Longrightarrow(2)$. According to Theorem 2.1 it suffices to construct a $P$-invertible map $f_{P}: K_{P} \rightarrow \mathbb{I}^{\omega}$ where $K_{P}$ is a metrizable compactum with $P \in$ $A E\left(K_{P}\right)$. Since $P$ is finitely dominated, there exist a finite complex $L$ and two maps $u: P \rightarrow L$ and $d: L \rightarrow P$ such that $d u \simeq \operatorname{id}_{P}$. By [7, Theorem 2.3] there exist a compactum $K_{P}$ and a map $f_{P}: K_{P} \rightarrow \mathbb{I}^{\omega}$ with the following properties:

(a) For each map $g: Z \rightarrow \mathbb{I}^{\omega}$, defined on a separable metrizable space with $P \in$ $A E(Z)$, there exists a map $h: Z \rightarrow K_{P}$ such that $f_{P} h=g$.

(b) For each map $\varphi: C \rightarrow P$, where $C$ is a closed subset of $K_{P}$, there exists a map $\varphi^{\prime}: K_{P} \rightarrow L$ such that $\varphi^{\prime} \mid C \simeq u \varphi$.

Observe that, by (b), $d \varphi^{\prime} \mid C \simeq d u \varphi \simeq \varphi$. Consequently, by the Homotopy Extension Theorem, the map $\varphi$ has a continuous extension over $K_{P}$. This in turn means that $P \in A E\left(K_{P}\right)$. By (a) and [3, Proposition 5.2], the map $f_{P}$ is $P$-invertible.

Proposition 2.3. Let $P$ be a connected locally compact simplicial complex of finite type with a finite fundamental group. Then the following conditions are equivalent for any space $X$ and any integer $n \geq 2$ :

(a) $P \vee S^{n} \in A E(X)$.

(b) $P \vee S^{n} \in A E(\beta X)$.

Proof. The implication (b) $\Longrightarrow$ (a) follows from [3, Proposition 6.8]. 
In order to prove the implication (a) $\Longrightarrow$ (b) it suffices to show that $P \vee S^{n} \in$ $A E(\beta X)$ for each normal space $X$ with $P \vee S^{n} \in A E(X)$ (see Theorem 2.1(b)). Let $\varphi: F \rightarrow P \vee S^{n}$ be a map defined on a closed subset $F$ of $\beta X$. Since $P \vee S^{n}$ is an $A N R$, there exists an extension $\psi: \operatorname{cl}_{\beta X} V \rightarrow P \vee S^{n}$ of $\varphi$, where $V$ is an open neighborhood of $F$ in $\beta X$. Clearly $V \cap X \neq \emptyset$ and $\operatorname{cl}_{\beta X}\left(\operatorname{cl}_{X}(V \cap X)\right)=$ $\operatorname{cl}_{\beta X} V$. Since $P \vee S^{n} \in A E(X)$, there exists a map $f: X \rightarrow P \vee S^{n}$ such that $f\left|\operatorname{cl}_{X}(V \cap X)=\psi\right| \operatorname{cl}_{X}(V \cap X)$. An argument similar to 1, Proof of Lemma 4.1] shows that $f$ is homotopic to a map $g: X \rightarrow P \vee S^{n}$ such that $\operatorname{cl}(g(X))$ is compact. Consequently $g$ has a continuous extension $\bar{g}: \beta X \rightarrow P \vee S^{n}$ onto the whole $\beta X$. Now consider the two maps $\psi$ and $\bar{g} \mid \operatorname{cl}_{\beta X} V$. Their restrictions $\psi \mid \operatorname{cl}_{X}(V \cap X)$ and $\bar{g} \mid \operatorname{cl}_{X}(V \cap X)$ are homotopic. Since $X$ is normal, it follows that $\operatorname{cl}_{\beta X} V=\operatorname{cl}_{\beta X}\left(\operatorname{cl}_{X}(V \cap X)\right)=\beta\left(\operatorname{cl}_{X}(V \cap X)\right)$. By [1, Theorem 4.2], the restriction operator provides a bijection of homotopy classes $\left[\beta\left(\operatorname{cl}_{X}(V \cap X)\right), P \vee S^{n}\right]$ and $\left[\mathrm{cl}_{X}(V \cap X), P \vee S^{n}\right]$. Consequently, $\psi \simeq \bar{g} \mid \operatorname{cl}_{\beta X} V$. By the homotopy extension theorem (recall that $\bar{g} \mid \operatorname{cl}_{\beta X} V$ has an extension $\bar{g}$ onto $\beta X$ and that $P \vee S^{n}$ is an $A N R), \psi$ also has an extension onto $\beta X$ which serves as an extension of the originally given map $\varphi$.

Finally I would like to thank the referee for helpful suggestions which led to a number of improvements of the original version of these notes.

\section{REFERENCES}

[1] A. Calder, J. Siegel, Homotopy and uniform homotopy, Trans. Amer. Math. Soc. 235 (1978), 245-270. MR 56:16619

[2] A. Chigogidze, Inverse Spectra, North Holland, Amsterdam, 1996. MR 97g:54001

[3] A. Chigogidze, Cohomological dimension of Tychonov spaces, Topology Appl. 79 (1997), 197-228. CMP 97:17

[4] A. Chigogidze, Infinite dimensional topology and Shape theory, in: Handbook of Geometric Topology, ed. by R. Daverman and R. Sher (to appear).

[5] A. N. Dranishnikov, Cohomological dimension is not preserved by Stone-Čech compactification, Comptes Rendus Bulgarian Acad. Sci. 41 (1988), 9-10. MR 90e:55002

[6] A. N. Dranishnikov, J. Dydak, Extension dimension and extension types, Proc. Steklov Math. Inst. 212 (1996), 55-88. CMP 98:16

[7] J. Dydak, Cohomological dimension of metrizable spaces. II, Trans. Amer. Math. Soc. 348 (1996), 1647-1661. MR 96h:55001

[8] J. Dydak, J. Mogilski, Universal cell-like maps, Proc. Amer. Math. Soc. 122 (1994), 943-948. MR 95a:55003

[9] J. Dydak, J. J. Walsh, Spaces without cohomological dimension preserving compactifications, Proc. Amer. Math. Soc. 113 (1991), 1155-1162. MR 92c:54039

[10] A. I. Karinski, On cohomological dimension of the Stone-Čech compactification, Vestnik Moscow Univ. no. 4 (1991), 8-11 (in Russian). MR 94a:55002

Department of Mathematics and Statistics, University of Saskatchewan, McLean Hall, 106 Wiggins Road, Saskatoon, Saskatchewan, Canada S7N 5E6

E-mail address: chigogid@math.usask.ca 